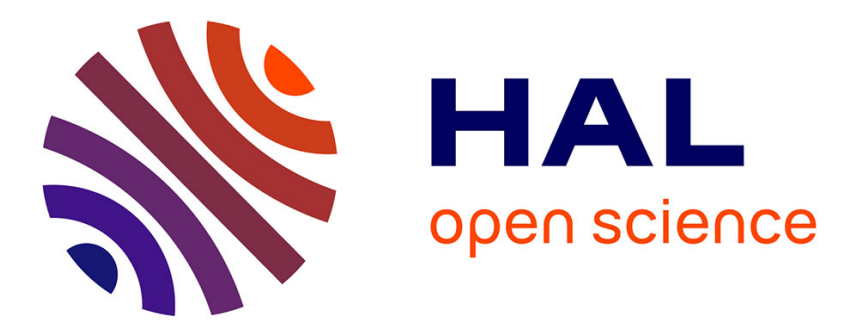

\title{
Stability of convection in a horizontal channel subjected to a longitudinal temperature gradient. Part 1. Effect of aspect ratio and Prandtl number
}

T.P. Lyubimova, D.V. Lyubimov, V. A. Morozov, R.V. Scuridin, Hamda Ben Hadid, Daniel Henry

\section{To cite this version:}

T.P. Lyubimova, D.V. Lyubimov, V. A. Morozov, R.V. Scuridin, Hamda Ben Hadid, et al.. Stability of convection in a horizontal channel subjected to a longitudinal temperature gradient. Part 1. Effect of aspect ratio and Prandtl number. Journal of Fluid Mechanics, 2009, 635, pp.275-295. 10.1017/S0022112009007587 . hal-00441920

\author{
HAL Id: hal-00441920 \\ https://hal.science/hal-00441920
}

Submitted on 17 Dec 2009

HAL is a multi-disciplinary open access archive for the deposit and dissemination of scientific research documents, whether they are published or not. The documents may come from teaching and research institutions in France or abroad, or from public or private research centers.
L'archive ouverte pluridisciplinaire HAL, est destinée au dépôt et à la diffusion de documents scientifiques de niveau recherche, publiés ou non, émanant des établissements d'enseignement et de recherche français ou étrangers, des laboratoires publics ou privés. 


\title{
Stability of convection in a horizontal channel subjected to a longitudinal temperature gradient. Part 1. Effect of aspect ratio and Prandtl number
}

\author{
T. P. LYUBIMOVA ${ }^{1}$, D. V. LYUBIMOV ${ }^{2}$, V. A. MOROZOV ${ }^{3}$, \\ R. V. SCURIDIN ${ }^{1}$, H. BEN HADID ${ }^{4} \dagger$ AND D. HENRY 4 \\ ${ }^{1}$ Institute of Continuous Media Mechanics UB RAS, 1 Koroleva Street, 614013 Perm, Russia \\ ${ }^{2}$ Perm State University, 15 Bukirev Street, 614990 Perm, Russia \\ ${ }^{3}$ Argonne National Laboratory, 9700 South Cass Avenue, Argonne, IL 60439, USA \\ ${ }^{4}$ Université de Lyon, École Centrale de Lyon/Université Lyon 1/INSA de Lyon, Laboratoire de \\ Mécanique des Fluides et d'Acoustique, UMR-CNRS 5509, 43 Bd du 11 Novembre 1918, \\ 69622 Villeurbanne Cedex, France
}

(Received 20 December 2007; revised 30 March 2009; accepted 9 May 2009)

The paper deals with the numerical investigation of the steady convective flow in a horizontal channel of rectangular cross-section subjected to a uniform longitudinal temperature gradient imposed at the walls. It is shown that at zero Prandtl number the solution of the problem corresponds to a plane-parallel flow along the channel axis. In this case, the fluid moves in the direction of the imposed temperature gradient in the upper part of the channel and in the opposite direction in the lower part. At non-zero values of the Prandtl number, such solution does not exist. At any small values of $\mathrm{Pr}$ all three components of the flow velocity differ from zero and in the channel cross-section four vortices develop. The direction of these vortices is such that the fluid moves from the centre to the periphery in the vertical direction and returns to the centre in the horizontal direction. The stability of these convective flows (uniform along the channel axis) with regard to small three-dimensional perturbations periodical in the direction of the channel axis is studied. It is shown that at low values of the Prandtl number the basic state loses its stability due to the steady hydrodynamic mode related to the development of vortices at the boundary of the counter flows. The growth of the Prandtl number results in the strong stabilization of this instability mode and, beyond a certain value of the Prandtl number depending on the crosssection aspect ratio, a new steady hydrodynamic instability mode becomes the most dangerous. This mode is characterized by the localization of the perturbations near the sidewalls of the channel. At still higher values of the Prandtl number, the spiral perturbations (rolls with axis parallel to the temperature gradient) become the most dangerous modes, at first the oscillatory spiral perturbations and then the Rayleightype steady spiral perturbations. The influence of the channel width on these different instabilities is also emphasized.

\section{Introduction}

A great amount of microelectronic and opto-electronic devices are manufactured by direct solidification of molten material. During the growth processes the molten

$\dagger$ Email address for correspondence: hamda.benhadid@univ-lyon1.fr 
material is subject to varying heat- and mass-transfer conditions which, in turn, profoundly affect the microscopic composition of the melt-grown crystals (see, e.g. Carruthers 1977). It is therefore necessary to understand the underlying physical mechanisms determining the fluid dynamics and its stability. In many cases, preventing the primary instability (the first bifurcation of the flow) would mean stabilizing the process as a whole, which is extremely desirable for various crystal growth technologies.

Confined shallow cavity heated from a side is one of the models for the horizontal Bridgman configuration, a popular type of melt-growth process. In this system, the temperature gradient is orthogonal to the gravity, and then convection arises for any value of the temperature difference. This configuration is the focus of this study, and the transitions in a channel flow subject to a uniform temperature gradient are expected to qualitatively reproduce the scenario of appearance of the instabilities in the melt flow for such a growth method.

The Bridgman configuration has been mathematically modelled by numerous researchers. The models couple the effects of transient fluid dynamics and energy and mass transfer within the system. Over the years, the studies concerned analytical (Cormack, Leal \& Imberger 1974; Gill 1974), as well as numerical linear stability analyses for the one-dimensional velocity and temperature profiles (Hart 1972, 1983; Gershuni, Zhukhovitskii \& Myznikov 1974a,b; Laure 1987; Laure \& Roux 1987; Kuo \& Korpela 1988). Numerous workers (Winters 1988; Roux, Ben Hadid \& Laure 1989a, b; Skeldon, Riley \& Cliffe 1996; Gelfgat, Bar-Yoseph \& Yarin 1999) stimulated by the GAMM workshop (Roux 1990) carried out two-dimensional numerical simulations, and more recently, results from three-dimensional numerical simulations were given by Henry \& Buffat (1998), Wakitani (2001) and Henry \& Ben Hadid $(2007 a, b)$ These works addressed the effect of the main parameters, the Grashof number and the cavity aspect ratios (length/height and width/height), on the structure and stability of the low-Prandtl-number flows. A main feature of the results is that the critical Grashof number for the occurrence of time-dependent flows is strongly dependent on the Prandtl number and the cavity aspect ratios.

The linear stability of the base state in the infinite extent horizontal layer has been an active area of research since the early work of Gill (1974), but there is now a clear agreement between the results of the different studies. The one cell circulation (Hadley circulation) loses its stability when the Grashof number exceeds some critical value which is found to depend largely on the Prandtl number. Three perturbing mechanisms are found to occur for rigid conducting boundaries: two-dimensional steady hydrodynamic modes, three-dimensional oscillatory spiral waves and threedimensional Rayleigh modes. The hydrodynamic mode which corresponds to a shear instability is the most dangerous for $\mathrm{Pr}<0.14$ and generates steady rolls with the axis perpendicular to the temperature gradient. The oscillatory spiral mode which occurs in a narrow range of Prandtl numbers $0.14<\operatorname{Pr}<0.44$, corresponds to the development of internal waves and leads to the formation of oscillatory rolls with the axis parallel to the temperature gradient. The potentially unstable temperature stratification created by the basic flow near the upper and lower horizontal boundaries, makes possible the appearance of the Rayleigh-Bénard like instability for large values of the Grashof number. This type of instability prevails for large Prandtl numbers $\operatorname{Pr}>0.44$, and does not exist in the case of insulated horizontal boundaries. In this last case, the hydrodynamic modes prevail for $\mathrm{Pr}<0.033$ and the oscillatory three-dimensional spiral modes become dominant for $0.033<\operatorname{Pr}<0.2$.

Concerning the experiments, the main focus has been on time-dependent phenomena and there appear to be only few detailed experimental studies in the 
published literature. The pioneer work of Hurle, Jakeman \& Johnson (1974) reports measurements of the motion in a cavity with small aspect ratios $2.73 \times 1.18 \times 1$ (length $\times$ width $\times$ height) and finds that at moderate Grashof number the flow is three-dimensional in nature, and is symmetric with respect to the vertical longitudinal midplane of the cavity. In contrast Hung \& Andereck (1990) used a cavity with large aspect ratios $(17.89 \times 17.78 \times 1)$ to generate an approximately two-dimensional flow. The main feature of their results is that the steady long-scale convective loop loses stability to a corotating multicell state. Braunsfurth et al. (1997) performed experiments in gallium in a cavity with moderate aspect ratios. They compare their observations from experimental study with the standard Hadley circulation solution and with the results of two-dimensional numerical calculations. They found a good agreement between the three approaches.

A small number of three-dimensional time-dependent calculations have been performed for differentially heated cavity. In crystal growth processes as well as in laboratory experiments, the flows are three-dimensional, and thus studies extending the purely two-dimensional approaches to take into account the three-dimensional features which develop not only near the endwalls but also in the core of the enclosure (Henry \& Buffat 1998) are needed. Most studies, following the GAMM workshop (Roux 1990), have considered the flow developing in the longitudinal vertical plane. To take into account the transverse variations of the flow in the core region of the enclosure, we derived a model which considers the three components of the velocity in the cross-section of an infinite length channel. Both steady state calculations and stability analyses with regard to fully three-dimensional perturbations are performed. To our knowledge, this is the first modelling study of this specific system. The relevant dimensionless numbers are the well-known Prandtl $(P r)$ and Grashof $(G r)$ numbers, and the transverse aspect ratio $l$ (width/height).

In this paper, we first determine the steady flow and temperature field representing the base state in the rectangular cross-section of a horizontal channel submitted to a uniform and longitudinal temperature gradient. We then perform a linear stability analysis based on the sensitivity of the base state to small three-dimensional perturbations periodical in the direction of the channel axis, and investigate the dependence of the first bifurcation and the perturbation structure on the transverse aspect ratio and the Prandtl number.

\section{Governing equations and boundary conditions}

We consider a flow in a horizontal channel with a rectangular cross-section of width $L$ and height $H$ (figure 1 ). An aspect ratio is defined by $l=L / H$. A uniform temperature gradient $(\nabla T)^{*}$ parallel to the channel axis is imposed on the channel walls. The flow arising in this situation is described by the conventional NavierStokes equations coupled to the energy equation. In the Boussinesq approximation, these equations are

$$
\begin{gathered}
\frac{\partial \boldsymbol{V}^{*}}{\partial t^{*}}+\left(\boldsymbol{V}^{*} \cdot \nabla^{*}\right) \boldsymbol{V}^{*}=-\frac{1}{\rho} \nabla^{*} P^{*}+v \nabla^{* 2} \boldsymbol{V}^{*}+g \beta T^{*} \boldsymbol{e}_{y}, \\
\frac{\partial T^{*}}{\partial t^{*}}+\left(\boldsymbol{V}^{*} \cdot \nabla^{*} T^{*}\right)=\kappa \nabla^{* 2} T^{*} \\
\nabla^{*} \cdot \boldsymbol{V}^{*}=0 .
\end{gathered}
$$

The boundary conditions are no-slip conditions and a fixed temperature distribution (purely conducting walls): $\left.\quad \boldsymbol{V}^{*}\right|_{\Gamma}=\left.\left(\vec{U}^{*}, W^{*}\right)\right|_{\Gamma}=0,\left.\quad T^{*}\right|_{\Gamma}=(\nabla T)^{*} z^{*}$. Here 


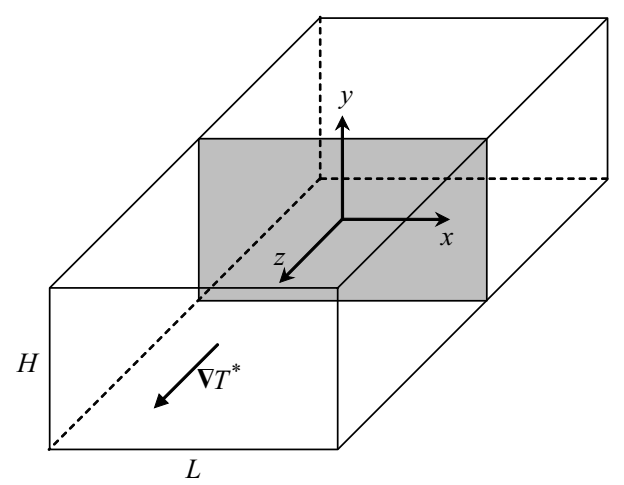

FIGURE 1. Studied configuration.

$\vec{U}^{*}=\left(U^{*}, V^{*}\right)$ is the transverse velocity, $W^{*}$ is the longitudinal velocity, $\boldsymbol{e}_{y}$ is the unit vector directed vertically upwards, $z^{*}$ is the coordinate in the direction of the channel axis and $\Gamma$ is the boundary of the channel cross-section $S$. The quantities with a star refer to dimensional quantities.

Using $H, H^{2} / v, v / H, \rho_{0} v^{2} / H^{2}$ and $(\nabla T)^{*} H$ as scales for length, time, velocity, pressure and temperature, respectively, the governing equations for the problem are

$$
\begin{gathered}
\frac{\partial \boldsymbol{V}}{\partial t}+(\boldsymbol{V} \cdot \nabla) \boldsymbol{V}=-\nabla P+\nabla^{2} \boldsymbol{V}+G r T \boldsymbol{e}_{y}, \\
\frac{\partial T}{\partial t}+(\boldsymbol{V} \cdot \nabla T)=\frac{1}{P r} \nabla^{2} T, \\
\nabla \cdot \boldsymbol{V}=0,
\end{gathered}
$$

where $\boldsymbol{V}=(\vec{U}, W)=(U, V, W), T, x, y$ and $z$ are the dimensionless variables, $P r=v / \kappa$ is the Prandtl number and $G r=g \beta(\nabla T)^{*} H^{4} / \nu^{2}$ is the Grashof number. The associated boundary conditions applied on $x= \pm l / 2$ and $y= \pm 1 / 2$ are $V=0$ and $T=T_{c}$, where $T_{c}$ is the conductive temperature.

\section{Basic flow}

\subsection{Basic flow equations}

We look for the basic flow solution in which the velocity and temperature deviations from the conductive state do not depend on $z$ :

$$
\vec{U}=\vec{U}(x, y), \quad W=W(x, y), \quad T=\Theta(x, y)+z .
$$

Equations (2.4)-(2.6) take the form:

$$
\begin{gathered}
\frac{\partial \vec{U}}{\partial t}+\left(\vec{U} \cdot \nabla_{s}\right) \vec{U}=-\nabla_{s} P+\nabla_{s}^{2} \vec{U}+G r(\Theta+z) \boldsymbol{e}_{y}, \\
\frac{\partial W}{\partial t}+\left(\vec{U} \cdot \nabla_{s} W\right)=-\frac{\partial P}{\partial z}+\nabla_{s}^{2} W, \\
\frac{\partial \Theta}{\partial t}+\left(\vec{U} \cdot \nabla_{s} \Theta\right)+W=\frac{1}{P r} \nabla_{s}^{2} \Theta, \\
\nabla_{s} \cdot \vec{U}=0,
\end{gathered}
$$


where $\nabla_{s}$ is the operator of differentiation in the plane $(x, y)$. It follows from (3.2) that $P$ is a linear function of $z\left(\partial^{2} P / \partial z^{2}=0\right)$, and we obtain from (3.1) that

$$
\nabla_{s} \frac{\partial P}{\partial z}=\operatorname{Gr} \boldsymbol{e}_{y} .
$$

Projecting (3.5) on the $x$ and $y$ axes, we obtain that

$$
P=\operatorname{Gr} y z+\Pi(x, y)+C z,
$$

where $C$ is a constant. Accounting for (3.6), we can rewrite (3.1)-(3.4) in the form:

$$
\begin{gathered}
\frac{\partial \vec{U}}{\partial t}+\left(\vec{U} \cdot \nabla_{s}\right) \vec{U}=-\nabla_{s} \Pi+\nabla_{s}^{2} \vec{U}+G r \Theta \boldsymbol{e}_{y}, \\
\frac{\partial W}{\partial t}+\left(\vec{U} \cdot \nabla_{s} W\right)=-G r y-C+\nabla_{s}^{2} W, \\
\frac{\partial \Theta}{\partial t}+\left(\vec{U} \cdot \nabla_{s} \Theta\right)+W=\frac{1}{P r} \nabla_{s}^{2} \Theta, \\
\nabla_{s} \cdot \vec{U}=0 .
\end{gathered}
$$

The constant $C$ is determined from mass conservation in the channel cross-section $S$, $\int_{S} W \mathrm{~d} S=0$, which gives:

$$
C=\frac{1}{S} \int_{S} \nabla_{s}^{2} W \mathrm{~d} S=\frac{1}{S} \oint_{\Gamma} \frac{\partial W}{\partial n} \mathrm{~d} \Gamma,
$$

where $n$ is the normal to the channel surface. For the transverse flow in the crosssection, it is convenient to introduce the stream function $\psi$ and the vorticity $\Omega$, which are such that $\vec{U}=\left(\nabla \times \psi \boldsymbol{e}_{z}\right),(\nabla \times \vec{U})_{z}=-\nabla_{s}^{2} \psi=\Omega$, where $\boldsymbol{e}_{z}$ is the unit vector in the direction of the channel axis.

\subsection{Theoretical developments}

In the general case, it can be shown that the problem under consideration does not allow the solutions which correspond to a plane-parallel flow. Indeed, for such a flow $\vec{U}=\psi=\Omega=0$ and, as one can see by applying the curl operator to (3.7), the temperature deviation from the conductive state $\Theta$ does not depend on $x$. Then, as follows from (3.9), $W$ does not depend on $x$ too and, due to the no-slip boundary condition, $W=0$ all over the volume, which is in contradiction with (3.8). In contrast to that, for $\operatorname{Pr}=0$, there exists a steady plane-parallel flow solution for which $\vec{U}, \psi$, $\Omega, \Theta$ and $C$ are equal to zero, and $W$ is determined by the problem:

$$
\nabla_{s}^{2} W=G r y,\left.\quad W\right|_{\Gamma}=0 .
$$

Thus, at $\operatorname{Pr}=0$ the problem is linear and the flow velocity is proportional to $G r$. W is also even with respect to the horizontal coordinate and odd with respect to the vertical coordinate (the origin of the coordinate system is located at the centre of the cross-section). In the lower part of the channel, the fluid moves in the direction of the imposed temperature gradient and in the upper part, it moves in the opposite direction.

To better understand the influence of the parameters on the steady flow, we can also derive some asymptotic expansions. First, for small but non-zero values of the Prandtl number, we can look for a steady solution in the form of series with respect to $\operatorname{Pr}$ :

$$
W=W_{0}+\operatorname{Pr} W_{1}+\cdots,
$$




$$
\begin{aligned}
& \Theta=\operatorname{Pr} \Theta_{1}+\cdots, \\
& \psi=\operatorname{Pr} \psi_{1}+\cdots, \\
& \Omega=\operatorname{Pr} \Omega_{1}+\cdots .
\end{aligned}
$$

At zero order, $W_{0}$ is the plane-parallel flow solution proportional to $G r$ obtained at $P r=0$. From the first-order expansion, we obtain:

$$
\begin{aligned}
& \nabla_{s}^{2} \Omega_{1}=-G r \frac{\partial \Theta_{1}}{\partial x}, \\
& \nabla_{s}^{2} W_{1}=\mathscr{J}\left(W_{0}, \psi_{1}\right), \\
& \nabla_{s}^{2} \Theta_{1}=W_{0},
\end{aligned}
$$

where $\mathscr{J}\left(q, q^{\prime}\right)=(\partial q / \partial x)\left(\partial q^{\prime} / \partial y\right)-(\partial q / \partial y)\left(\partial q^{\prime} / \partial x\right)$. We see that for small values of the Prandtl number, the transverse components of the velocity (related to $\Omega$ ) are of the first order with respect to $\mathrm{Pr}$ and proportional to $\mathrm{Gr}^{2}$. Concerning the longitudinal velocity $W$, we find that the first-order term $W_{1}$ is proportional to $\mathrm{Gr}^{3}$ so that the small Prandtl number correction with respect to $W_{0}$ still scales as $\operatorname{Pr} \mathrm{Gr}^{2}$. From these observations, we can conclude that the expansion in series with respect to $\operatorname{Pr}$ is of limited practical interest for the calculation of the steady solutions for $\operatorname{Pr} \neq 0$ as this expansion is only valid for not too large $G r$. We also see that the linearity of the solution with respect to $G r$ at $P r=0$ fails at the first order in $P r$.

Another asymptotic expansion can be obtained for finite $P r$, but small $G r$. In that case, the steady solution can be expressed in the form of series with respect to $G r$. It is thus found that the expansions for $W$ and $\Theta$ only contain odd powers of $\mathrm{Gr}$ and the expansion for $\vec{U}$ only even powers, i.e. the structure of the transverse flow does not depend on the direction of the imposed temperature gradient. For all orders of expansion, the constant $C$ turns out to be zero. Therefore, the solution has welldefined parities with respect to the coordinates, which for the direction $x$ corresponds to a symmetry with respect to the $x=0$ plane and for the direction $y$ to a symmetry with respect to the $y=0$ axis. Certainly this does not mean that the solutions not having such symmetries cannot exist. However, they are absent, at least for small enough $G r$.

\subsection{Basic flow solutions}

The basic flow equations (3.7)-(3.10) were discretized using a finite difference method with second-order spatial derivatives. Two different methods were used to calculate the steady-state solutions: either the system of equations was integrated in time until the steady state was reached, or the system of stationary equations (obtained by assuming the time derivatives to be zero) was solved by the Newton method. In the case $P r=0$, the problem is reduced to a single Poisson equation for the longitudinal velocity component $W$, which was solved using the iterative SOR (successive overrelaxation) method. Typical grids of $101 \times 101$ points for $l=1$ have been used to calculate the basic solutions (see $\S 4.1$ where global tests concerning threshold values are given). Solutions which have been obtained close to the thresholds for different values of $\mathrm{Pr}$ and $l$ are presented in the following.

For zero Prandtl number, the isolines of the longitudinal flow velocity for the channels with $l=1$ and $l=4$ are displayed in figures 2 and 3 . As expected, the fluid moves from the warmer area to the colder area in the upper part of the channel and in the opposite direction in the lower part. From the results for $l=4$ (figure 3), we see that the influence of the sidewalls is effective on each side on a horizontal distance of 


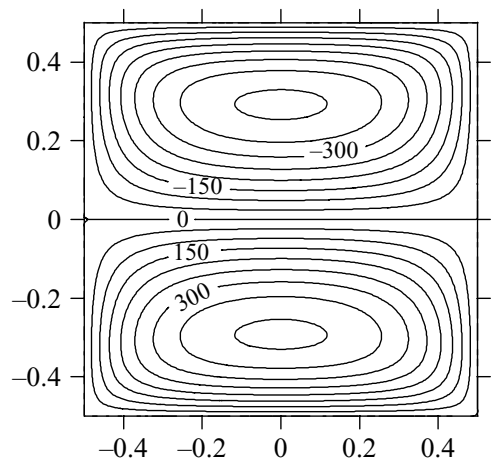

FIGURE 2. Isolines of the longitudinal component of the basic velocity at the critical threshold $\left(G r_{c}=55942\right)$ for $\operatorname{Pr}=0$ and $l=1$.

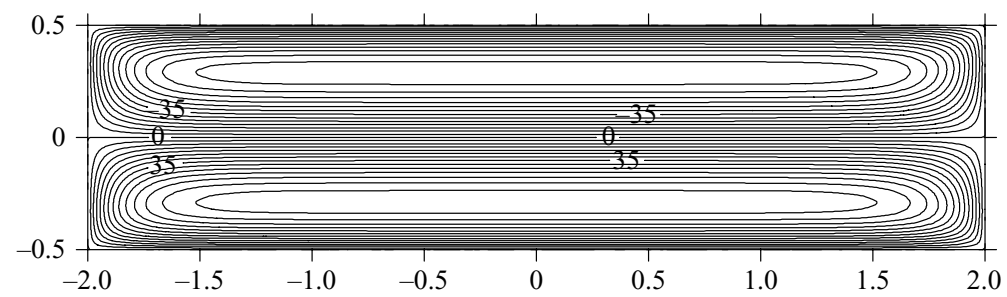

FIGURE 3. Isolines of the longitudinal component of the basic velocity at the critical threshold $\left(G r_{c}=8503\right)$ for $\operatorname{Pr}=0$ and $l=4$.

the order of the height of the channel. As a consequence, for $l=4$, there is a central zone in the channel where the velocity is independent of $x$ (determining identical vertical profiles of velocity similar to that obtained in the infinite layer), whereas for $l=1$ (figure 2) such zone is absent and the vertical profiles continuously change with $x$.

For non-zero Prandtl number values, the results are still presented for $l=1$ (figure 4 ) and $l=4$ (figure 5). For $l=1$, we see that the longitudinal flow leads to the appearance of a warm layer in the upper part of the channel and a cold layer in the lower part, whereas at the sidewalls the temperature remains the same (figure $4 c$ ). Thus, a difference in the temperatures of the central part of the channel and the sidewalls arises. As a result, an ascending flow is formed in the centre of the upper part of the channel and a descending flow in the centre of the lower part, which leads to the development of a transverse four-vortex flow in the channel (figure $4 a$ ). The vortex direction is such that the fluid moves from the centre to the periphery in the vertical direction and returns to the centre in the horizontal direction. The intensity of the transverse flow is proportional to $\mathrm{Pr}$, and at large enough values of the Prandtl number this flow leads to the modification of the longitudinal flow (figure $4 b$ ). Similar results are obtained for $l=4$. From figure 5(a) where the streamlines of the transverse flow are plotted, we can see that the transverse flow really arises due to the sidewall effect as the vortex centres are localized close to the sidewalls. As a consequence, the modification of the longitudinal flow by the transverse flow also occurs near the sidewalls (figure $5 b$ ). 

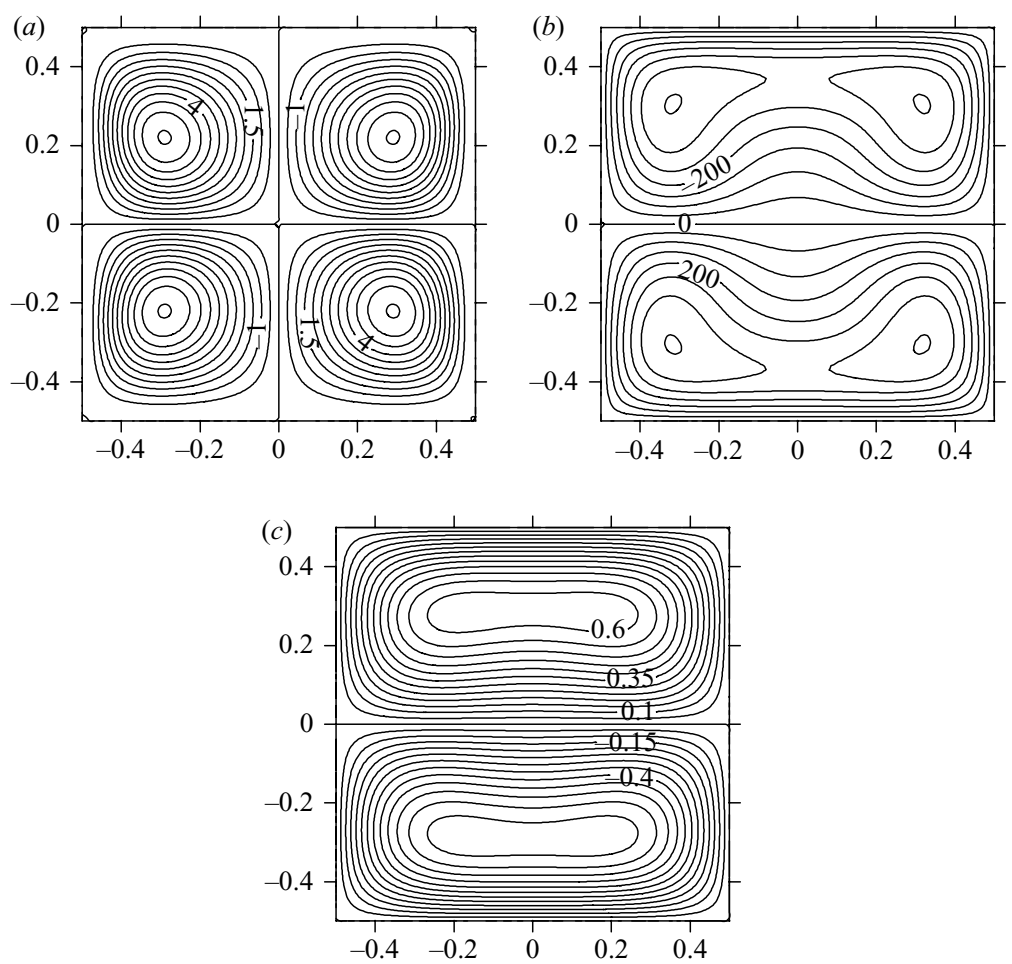

FIGURE 4. Streamlines of the basic transverse flow $(a)$, isolines of the basic longitudinal flow velocity $(b)$ and isolines of the basic temperature field $(c)$ at the critical threshold $\left(G r_{c}=66571\right)$ for $\operatorname{Pr}=0.1$ and $l=1$.

\section{Stability of the basic flow}

\subsection{Perturbation equation problem}

We now consider the stability of the convective flows obtained numerically in the previous section. To study the stability of these steady flows which are uniform in the direction of the channel axis, we analyse the behaviour of small normal-mode perturbations periodic in the $z$ direction:

$$
\begin{gathered}
\boldsymbol{V}=\boldsymbol{V}_{0}+\boldsymbol{V}^{\prime}, \quad P=P_{0}+P^{\prime}, \quad T=T_{0}+\Theta^{\prime}, \\
\left(\boldsymbol{V}^{\prime}, P^{\prime}, \Theta^{\prime}\right)=(\vec{v}, p, \theta) \mathrm{e}^{\mathrm{i} k z+\lambda t},
\end{gathered}
$$

where $k$ is the wavenumber in the $z$ direction and $\lambda=\lambda_{r}+\mathrm{i} \lambda_{i}$ is a complex growth rate. The linearized system of equations which describes the temporal evolution of these perturbations has the following form:

$$
\begin{aligned}
\lambda u+U_{0} \frac{\partial u}{\partial x}+u \frac{\partial U_{0}}{\partial x}+V_{0} \frac{\partial u}{\partial y}+v \frac{\partial U_{0}}{\partial y}+\mathrm{i} k W_{0} u & =-\frac{\partial p}{\partial x}+\nabla_{s}^{2} u-k^{2} u, \\
\lambda v+U_{0} \frac{\partial v}{\partial x}+u \frac{\partial V_{0}}{\partial x}+V_{0} \frac{\partial v}{\partial y}+v \frac{\partial V_{0}}{\partial y}+\mathrm{i} k W_{0} v & =-\frac{\partial p}{\partial y}+\nabla_{s}^{2} v-k^{2} v+G r \theta, \\
\lambda w+U_{0} \frac{\partial w}{\partial x}+u \frac{\partial W_{0}}{\partial x}+V_{0} \frac{\partial w}{\partial y}+v \frac{\partial W_{0}}{\partial y}+\mathrm{i} k W_{0} w & =-\mathrm{i} k p+\nabla_{s}^{2} w-k^{2} w,
\end{aligned}
$$


(a)

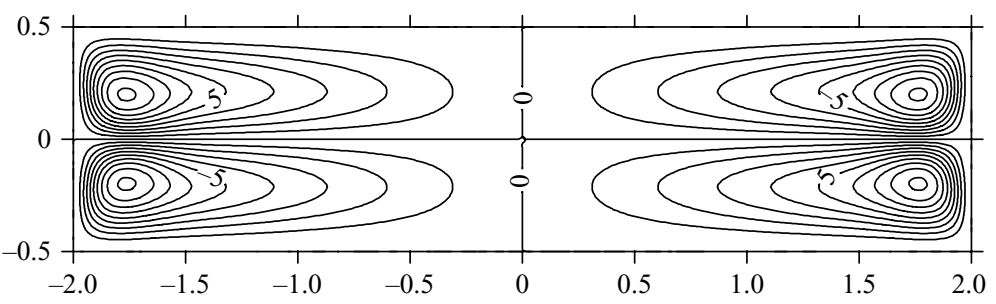

(b)

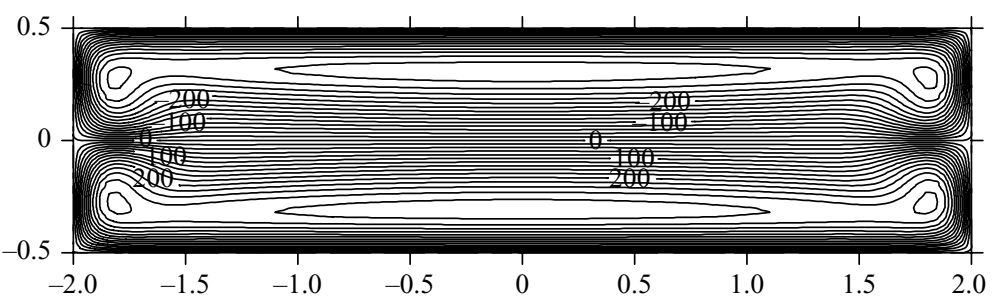

(c)

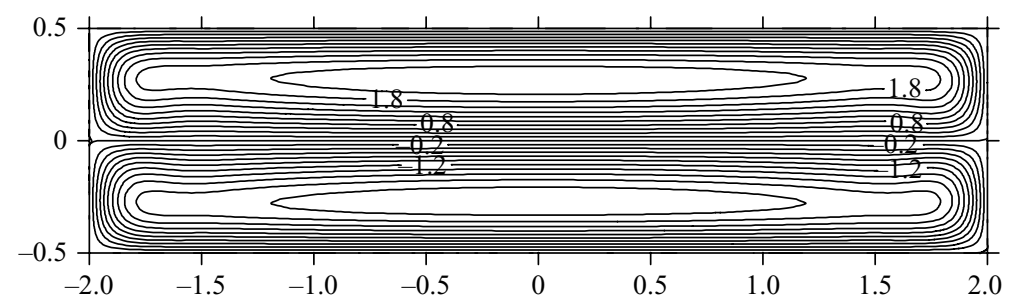

FiguRE 5. Streamlines of the basic transverse flow $(a)$, isolines of the basic longitudinal flow velocity $(b)$ and isolines of the basic temperature field $(c)$ at the critical threshold $\left(G r_{c}=40029\right)$ for $\operatorname{Pr}=0.308$ and $l=4$.

$$
\begin{aligned}
\lambda \theta+U_{0} \frac{\partial \theta}{\partial x}+u \frac{\partial T_{0}}{\partial x}+V_{0} \frac{\partial \theta}{\partial y}+v \frac{\partial T_{0}}{\partial y}+\mathrm{i} k W_{0} \theta+w & =\frac{1}{\operatorname{Pr}} \nabla_{s}^{2} \theta-\frac{k^{2}}{P r} \theta, \\
\frac{\partial u}{\partial x}+\frac{\partial v}{\partial y}+\mathrm{i} k w & =0 .
\end{aligned}
$$

Here $U_{0}, V_{0}, W_{0}$ and $T_{0}$ are the velocity components and the temperature of the basic flow, $u, v$ and $w$ are respectively the horizontal, vertical and longitudinal components of the velocity perturbations, $\theta$ is the temperature perturbation and $p$ is the pressure perturbation. The perturbations of velocity and temperature vanish at the boundaries. As follows from (4.6), at $P r=0$ the temperature perturbations vanish and the term responsible for the buoyancy force in (4.4) is absent.

The discretization of the perturbation equations by a finite difference method leads to the generalized algebraic eigenvalue problem $\boldsymbol{A} \boldsymbol{X}=\lambda \boldsymbol{B} \boldsymbol{X}$, where the matrix $\boldsymbol{A}$ is complex and has a band structure, the matrix $\boldsymbol{B}$ is diagonal, with part of the diagonal elements equal to unity and the others equal to zero, and $\boldsymbol{X}$ is the vector of the unknown perturbations. To solve this problem and thus obtain the eigenvalues and the corresponding eigenvectors (fields of velocity, temperature and pressure perturbations), a Newton-Raphson method is used. This method, however, needs to have a first guess of the eigenvalue and eigenvector which are looked 


\begin{tabular}{cccccc} 
& \multicolumn{2}{c}{$P r=0$} & & \multicolumn{2}{c}{$P r=0.1$} \\
\cline { 2 - 3 } \cline { 5 - 6 }$N_{x} \times N_{y}$ & $G r_{c}$ & $k_{c}$ & & $G r_{c}$ & $k_{c}$ \\
$45 \times 45$ & 64365 & 1.1091 & & 69127 & 1.2448 \\
$65 \times 65$ & 58437 & 1.1791 & & 67438 & 1.2754 \\
$83 \times 83$ & 56767 & 1.2004 & & 66868 & 1.2863 \\
$101 \times 101$ & 55942 & 1.2114 & & 66571 & 1.2919 \\
Spectral method & 55930 & 1.2233 & & 66183 & 1.3007 \\
$31 \times 31$ & & & &
\end{tabular}

TABLE 1. Variations of the critical Grashof number $G r_{c}$ and the associated critical wavenumber $k_{c}$ of the first steady bifurcation at various grid resolutions, for $l=1$ and two values of the Prandtl number, $P r=0$ and 0.1 .

for. This first guess is obtained through a direct numerical simulation based on the time evolution of the unsteady linearized equations. Starting from some initial state, the computation is continued until the evolution of the perturbation in time is only determined by the most dangerous mode. It is this mode, together with the eigenvalue and frequency which can be deduced from the final time evolution, which are introduced in the Newton-Raphson method. Finally, neutral perturbations are then obtained from the condition $\mathscr{R} e(\lambda)=0$, where $\mathscr{R} e$ denotes the real part. For more details on the methods, see the paper by Lyubimov, Lyubimova \& Morozov (2001) where the software package including these methods is presented.

To check the grid convergence, calculations with four different meshes were performed for $l=1$ and two Prandtl numbers, $P r=0$ and 0.1 . The results, given by the critical Grashof number and the critical wavenumber are summarized in table 1. All these grids give the same steady instability, but the variations of the critical Grashof number and the critical wavenumber indicate a small-grid dependence. However, the results of the finest grid $101 \times 101$ agree well with those obtained with a second code based on a spectral Galerkin method using a $31 \times 31$ Gauss-Lobatto-Legendre point distribution (Touihri, Ben Hadid \& Henry 1999; Vaux, Ben Hadid \& Henry 2006). The grid $101 \times 101$ was then estimated to be sufficient to give accurate solutions for $l=1$. Such grid was chosen to perform the calculations for $l=1$ reported in this work, and the grid was further refined in the $x$ direction for larger $l$.

\subsection{Fluctuating kinetic energy equation}

In order to get some physical insight into the stability results, it is often interesting to perform energy analyses (Kaddeche, Henry \& Ben Hadid 2003; Henry et al. 2008). For that, by multiplying the linear stability equations (4.3)-(4.5) by $\vec{v}^{*}$ (the complex conjugate of the velocity perturbation) and after integration on the cross-section $S$ and some simplifications, an equation giving the rate of change of the fluctuating kinetic energy can be derived. From this equation, for any instabilities at their critical thresholds $(\mathscr{R} e(\lambda)=0)$, normalized kinetic energy budgets can be obtained which we can write as

$$
E_{s}^{\prime}+E_{b}^{\prime}=1
$$

where $E_{s}^{\prime}=-\mathscr{R} e\left(\Sigma_{i=1}^{3} \Sigma_{j=1}^{2} \int_{S} v_{j} \frac{\partial V_{0_{i}}}{\partial x_{j}} v_{i}^{*} \mathrm{~d} x \mathrm{~d} y\right) /\left|E_{d}\right|$ are the productions of fluctuating kinetic energy by shear of the basic flow and $E_{b}^{\prime}=\mathscr{R} e\left(\int_{S} \operatorname{Gr} \theta v^{*} \mathrm{~d} x \mathrm{~d} y\right) /\left|E_{d}\right|$ is the production of fluctuating kinetic energy by buoyancy, all these terms being normalized by the viscous dissipation of fluctuating kinetic energy, $E_{d}=\mathscr{R} e\left(\Sigma_{i=1}^{3} \Sigma_{j=1}^{3}\right.$ 


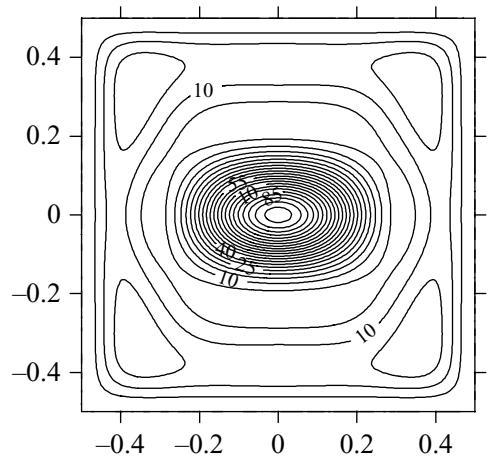

FIGURE 6. Isolines of the perturbation kinetic energy averaged in the $z$ direction at the critical threshold $\left(G r_{c}=55942\right)$ for $\operatorname{Pr}=0$ and $l=1$.

$\left.\int_{S} \frac{\partial^{2} v_{i}}{\partial x_{j}^{2}} v_{i}^{*} \mathrm{~d} x \mathrm{~d} y\right)$. In these expressions we have denoted the basic flow $\left[U_{0}, V_{0}, W_{0}\right]$ $(x, y, z)$ by $V_{0_{i}}\left(x_{i}\right)$ and the perturbations $[u, v, w](x, y, z)$ by $v_{i}\left(x_{i}\right)$.

One important shear term is generally the term related to the vertical variation of the basic longitudinal velocity, $E_{s y}^{\prime}=-\mathscr{R} e\left(\int_{S} v \frac{\partial W_{0}}{\partial y} w^{*} \mathrm{~d} x \mathrm{~d} y\right) /\left|E_{d}\right|$, which is the only term in the case of the infinite layer (Kaddeche et al. 2003). This term is obtained by integration of the spatial field $S_{y}=\mathscr{R} e\left(-\frac{\partial W_{0}}{\partial y} v w^{*}\right) /\left|E_{d}\right|$, which can itself be decomposed into two terms, $M=\left(-\frac{\partial W_{0}}{\partial y}\right)$ and $F=\mathscr{R} e\left(v w^{*}\right) /\left|E_{d}\right|$, respectively related to the basic flow and the fluctuating flow $\left[E_{s y}^{\prime}=\left(\int_{S} S_{y} \mathrm{~d} x \mathrm{~d} y\right)=\left(\int_{S} M F \mathrm{~d} x \mathrm{~d} y\right)\right]$.

\section{Stability results}

\subsection{Zero Prandtl number}

In the zero Prandtl number case, the temperature field is frozen and the instability is of hydrodynamic type; it is related to the development of vortices (with the axis parallel to $x$ ) at the boundary between the counter flows taking place in the upper and lower parts of the channel. As one can see from figure 6 where the isolines of the kinetic energy of the perturbations at threshold averaged in the $z$ direction are plotted for $l=1$, the kinetic energy of the perturbations is maximal at the channel axis.

The neutral curves $\operatorname{Gr}(k)$ for the case $l=1$ at $P r=0$ are plotted in figure 7. As one can see, the most dangerous perturbations are steady and they keep the symmetry of the basic flow with respect to the $x=0$ plane. The variations of the critical Grashof number and wavenumber of the most dangerous perturbations with the transverse aspect ratio $l$ for zero Prandtl number are shown in figure 8. It is found that the decrease of the transverse aspect ratio $l$ substantially stabilizes the flow and the perturbations with larger wavelength become the most dangerous. With the increase of the aspect ratio, the critical parameters asymptotically approach the values obtained in the case of the infinite layer (infinite extent in both $x$ and $z$ directions).

\subsection{Non-zero Prandtl number}

\subsubsection{Hydrodynamic instability mode}

We now consider the case of non-zero values of the Prandtl number. The results show that the steady hydrodynamic instability mode, which is the only instability mechanism for $\operatorname{Pr}=0$, still remains the most dangerous for small non-zero values of the Prandtl number. The dependence of the critical Grashof number on the Prandtl 


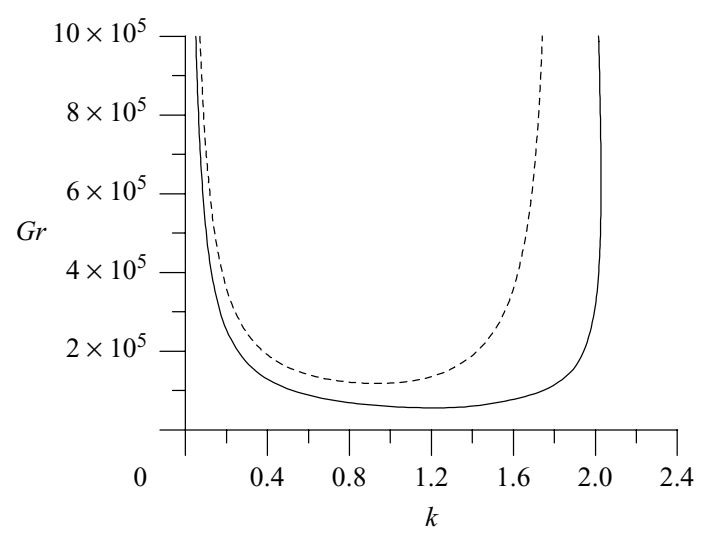

FIgURE 7. Neutral curves $\operatorname{Gr}(k)$ for $\operatorname{Pr}=0$ and $l=1$. Solid line: steady mode keeping the symmetry with respect to the $x=0$ plane; dashed line: steady mode breaking this symmetry.

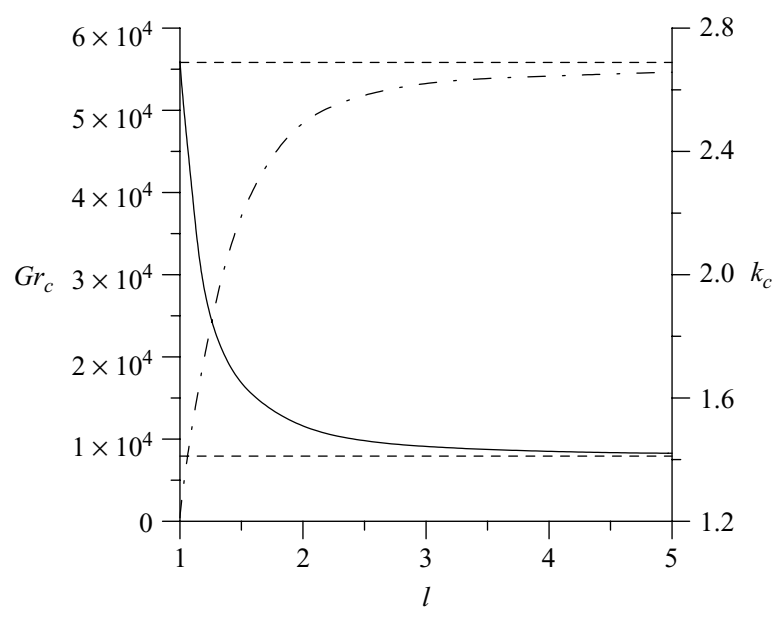

FIGURE 8. Critical Grashof number $G r_{c}$ (solid line) and wavenumber $k_{c}$ (dashed-dotted line) of the most dangerous perturbations versus the channel aspect ratio $l$ for $P r=0$. The dashed lines correspond to the critical values of the Grashof number and wavenumber for the case of the infinite horizontal layer.

number for this mode is shown in figure 9 for the channel with $l=4$. For comparison, we also plot the curve $\mathrm{Gr}_{c}(\mathrm{Pr})$ for the hydrodynamic mode in the case of the infinite layer $(l \rightarrow \infty)$. It is known that in the case of the infinite layer, a strong stabilization of the hydrodynamic instability mode is observed with the increase of the Prandtl number. This stabilization is related to the fact that at finite values of $\operatorname{Pr}$, a stable temperature stratification is created at mid-height of the cavity (figure $5 c$ ) in the domain where the perturbation vortices are triggered, which makes more difficult the vortices development. The curve $G r_{c}(P r)$ obtained for $l=4$ is characterized by two distinct portions of curves marked in figure 9 as $A B$ and $B C$. The value of $\operatorname{Pr}$ which corresponds to the transition between these two portions of curves is denoted as $\operatorname{Pr}_{t}$.

In order to understand the changes which occur at $P r_{t}$, an energy analysis is performed for three values of $\operatorname{Pr}(\operatorname{Pr}=0.10665,0.1067$ and 0.11$)$, close below and above $P r_{t}$. For the three cases, it is found that the destabilization comes from the 


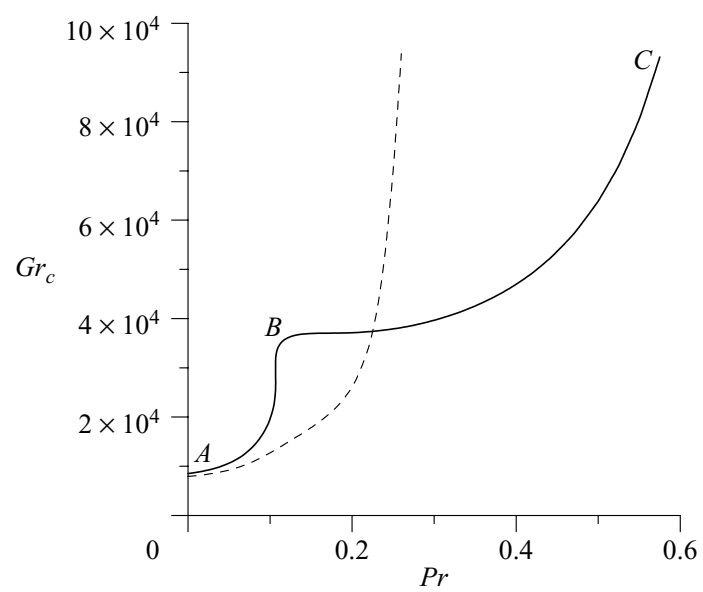

FIGURE 9. Critical Grashof number $G r_{c}$ for the hydrodynamic modes versus the Prandtl number $\operatorname{Pr}$ for $l=4$ (solid line) and the infinite horizontal layer (dashed line). $A B$ : steady hydrodynamic mode; $B C$ : steady sidewall hydrodynamic mode.

shear of the basic flow and is related to the term $E_{s y}^{\prime}$ which dominates all the other shear energy terms (by at least a factor 30), whereas buoyancy is stabilizing. The analysis of this dominant shear energy term is presented in figure 10 through the plots of the shear energy field $S_{y}$ and its two components $M$ and $F$. As it is clear from figure $10(a, d)$ where the plots are given for $\operatorname{Pr}=0.10665$ (close below $\operatorname{Pr}_{t}$ ), for the part $A B$ of the curve the mechanism of instability is the same as in the case of the infinite layer, with a destabilization due to shear at the boundaries between the counter flows in the central part of the cavity. The part $B C$ of the curve corresponds to a different instability mechanism. The destabilization is still related to the shear which exists at the boundary between the counter flows, but now the vortices located near the sidewalls (figure $5 a$ ) appear to modify the repartition of shear and to induce a change in the development of the perturbations. Indeed, the plots of $S_{y}, M$ and $F$ displayed in figures $10(b, e)$ and $10(c, f)$ for $P r=0.1067$ and 0.11 , respectively, show that two peaks appear in the shear energy field near the sidewalls in the regions where the counter-rotating vortices interact whereas the contribution in the central part of the cavity decreases, so that for $\mathrm{Pr}=0.11$ the contribution of the two shear energy peaks is largely dominant. Moreover, the plots of $M$ and $F$ reveal that the increase of the two peak contribution is connected to the shear $M$ which becomes slightly dominant near the sidewalls, but is dominated by the concomitant rapid growth of the velocity disturbances $F$ in this sidewall region. This sidewall shear instability is absent in the case of the infinite layer. As it is clear from figure 9, with further growth of the Prandtl number the sidewall shear instability is strongly stabilized too. The reason for this stabilization is the same as for the usual hydrodynamic instability existing in the case of the infinite layer.

The curves $G r_{c}(P r)$ and $k_{c}(P r)$ for the channel with $l=4$ are displayed in figure 11 for $\operatorname{Pr}<0.2$, in the domain of Prandtl number where the transition from the usual hydrodynamic mode to the sidewall instability mode occurs. We see that the transition is accompanied by a sharp decrease of the wavenumber which reaches a minimum and then starts to grow.

Figure 12 illustrates the effect of the decrease of the cross-section width $l$ on the steady flow destabilization by the hydrodynamic modes. As one would expect, the 


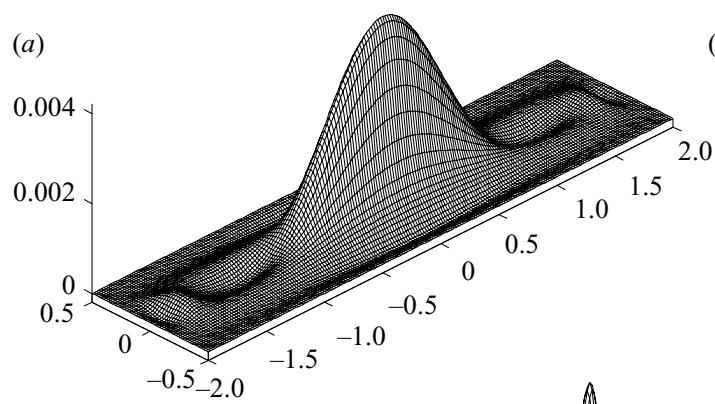

(d)
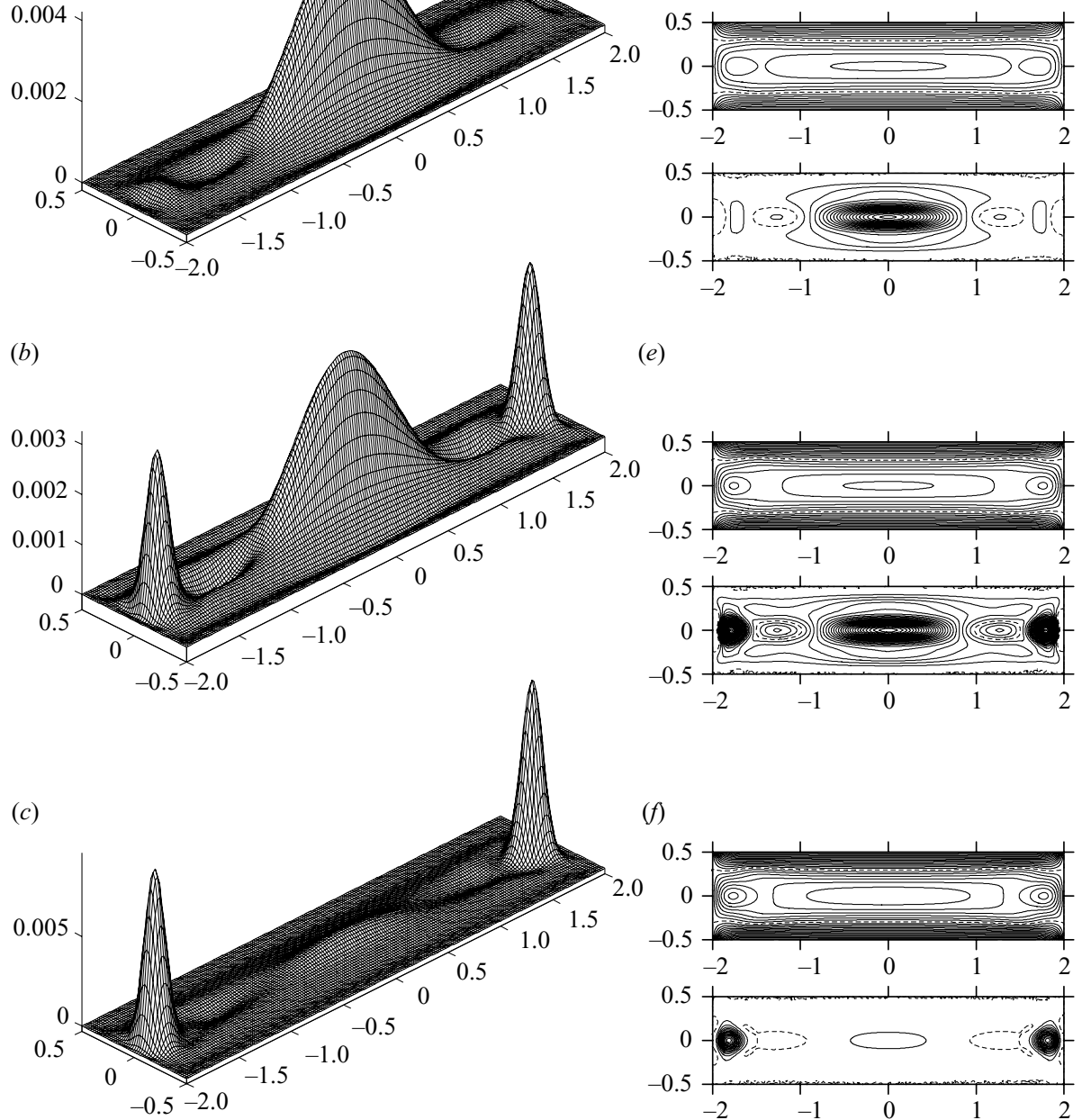

(e)

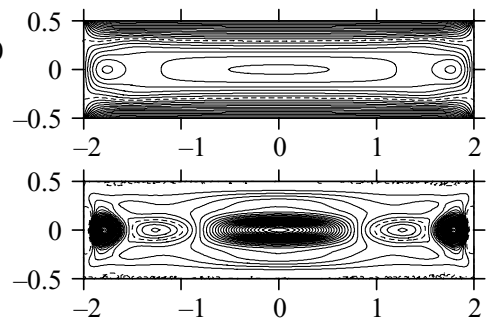

(f)

FIGURE 10. Three-dimensional views of the shear energy field $S_{y}(a-c)$ and isolines of its components $M$ (first plot) and $F$ (second plot) $(d-f)$ in the cross-section $S$, obtained from the basic flow and the perturbations at $G r_{c}$ for $l=4$ and three values of the Prandtl number, $\operatorname{Pr}=0.10665(a, d), \operatorname{Pr}=0.1067(b, e)$ and $\operatorname{Pr}=0.11(c, f)$. For $M$, the maximum isoline is 1000 for case $(d), 1200$ for case $(e)$ and 1400 for case $(f)$ and the step between isolines is 200 . For $F$, the maximum isoline is $4 \times 10^{-6}$ for $(d)\left(\right.$ step $\left.2 \times 10^{-7}\right), 2.6 \times 10^{-6}$ for $(e)$ (same maximum isoline in the central peak and in the lateral peaks) (step $1 \times 10^{-7}$ ) and $6.5 \times 10^{-6}$ for $(f)\left(\right.$ step $\left.5 \times 10^{-7}\right)$. In each picture, the zero isoline is given as a dashed line.

decrease of $l$ increases the sidewall influence, which results in the stabilization of the basic state (general increase of the critical thresholds) and in the earlier transition to the sidewall instability mode (smaller values of $P r_{t}$ ). If the induced modifications remain small when $l$ is changed from 4 to 3 , they become important for $l=1.5$ and really strong for $l=1$. In particular, it is worth to note the strong shift of the transition between the two hydrodynamic modes towards very small values of $\operatorname{Pr}\left(\operatorname{Pr}_{t} \approx 0.001\right.$ for $l=1$ ), which means that for strongly confined channels only the sidewall instability will be observable for usual low-Prandtl-number fluids. The evolution of the perturbation kinetic energy at threshold with the Prandtl number for $l=1$ is shown in figure 13 . 


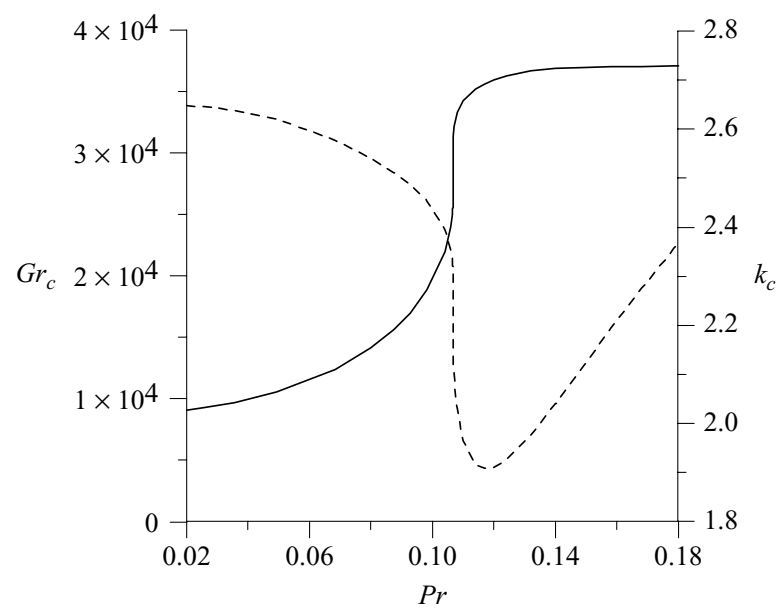

Figure 11. Curves $G r_{c}(P r)$ (solid line) and $k_{c}(P r)$ (dashed line) for $l=4$ in the Prandtl number range where the transition to the sidewall instability mode occurs.

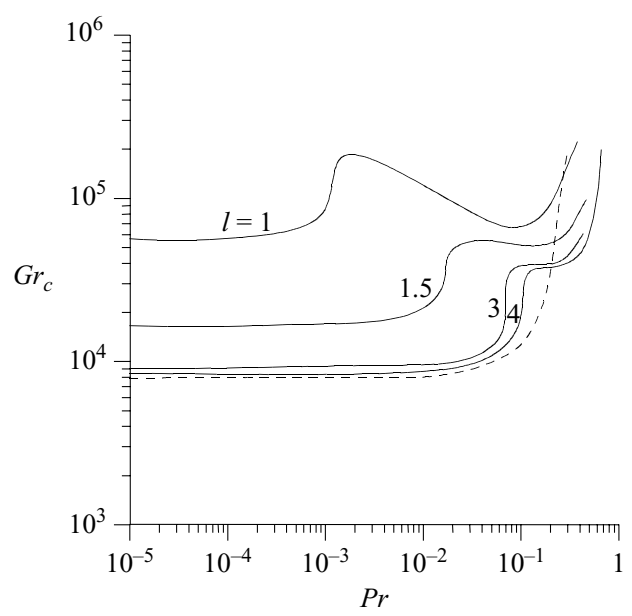

FIGURE 12. Critical Grashof number $G r_{c}$ for the hydrodynamic modes versus the Prandtl number $\operatorname{Pr}$ for different values of the channel aspect ratio $l$. The dashed line refers to the infinite horizontal layer.

We can see that the perturbation kinetic energy evolves progressively as the value of the Prandtl number is increased from one peak concentrated in the centre part of the cross-section to two peaks located near the sidewalls. This behaviour, obtained for a square cross-section cavity, corroborates what was already observed for a large extent cavity $(l=4)$ through an energy analysis (figure 10$)$.

We give a final remark related to fully three-dimensional cavities. From the studies of Henry \& Buffat (1998) and Henry \& Ben Hadid (2007a), we know that for not too small confinement ( $l$ around 2 or more), the steady solution in a side-heated cavity evolves with the increase of the Grashof number from a long-scale unicellular circulation to a roll-like structure. These authors mention that this evolution is the sign of an imperfect bifurcation which is connected to the steady transition (involving a hydrodynamic multi-roll mode) found at small $\mathrm{Pr}$ in the infinite layer. The present 

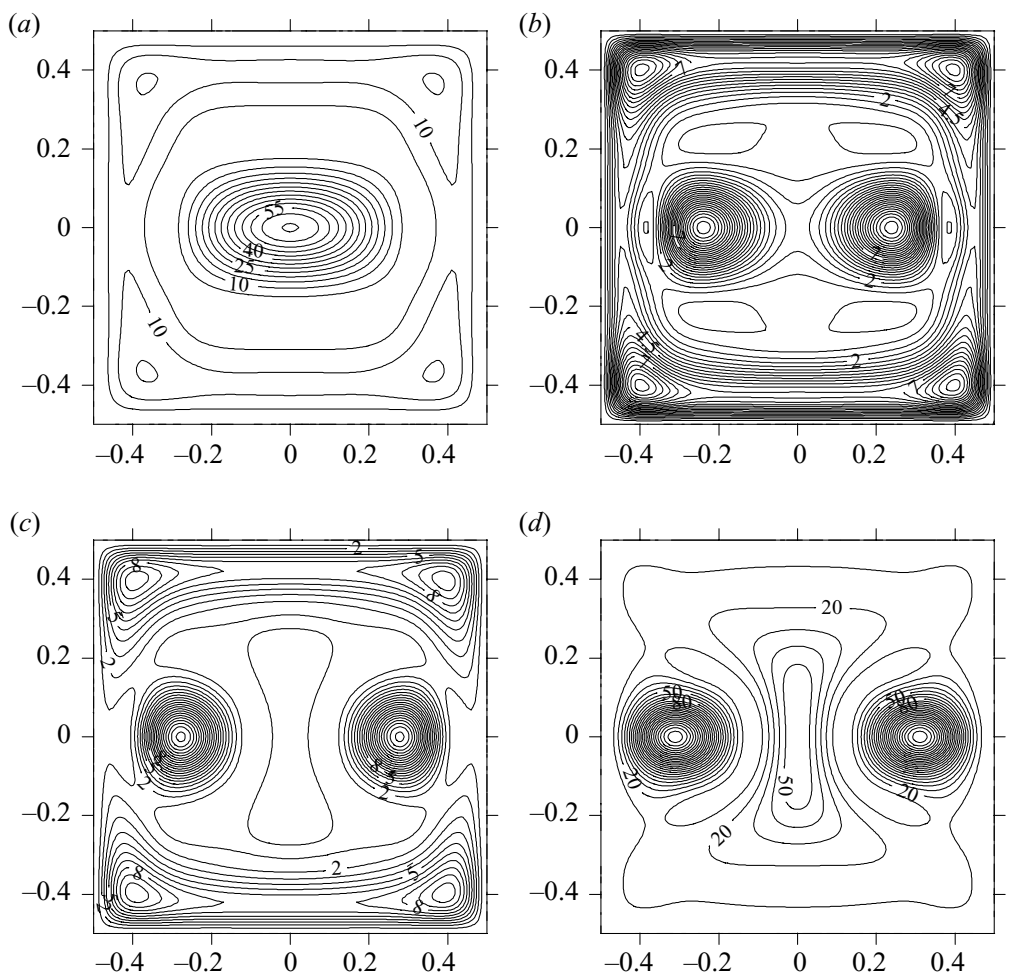

FIGURE 13. Isolines of the perturbation kinetic energy averaged in the $z$ direction for the hydrodynamic modes at the critical threshold in the case $l=1$ for different values of the Prandtl number: $\operatorname{Pr}=0.001(a), \operatorname{Pr}=0.002(b), \operatorname{Pr}=0.004(c), \operatorname{Pr}=0.1(d)$.

study indicates that similar steady transitions are found at small $\mathrm{Pr}$ for finite width channels. More precisely, from figure 12, we learn that for usual low-Prandtl-number values $(0.01 \leqslant P r \leqslant 0.026)$, this transition is related to the usual hydrodynamic mode for $l>1.5$, whereas for $l<1.5$ it is related to the sidewall hydrodynamic mode. This is consistent with the fact that the roll-like structure in the main circulation plane $(x=0)$ has been found numerically at $\operatorname{Pr}=0.01$ for $l=1.6$, but not for $l=1$ (Henry $\&$ Ben Hadid 2007a), and that this structure has not been found experimentally for $l=1.3$ at $P r \approx 0.02$ (Hof et al. 2004). Now, it would be interesting to more attentively analyse the flow structures in strongly confined cavities to see if signs of the sidewall instability could be found.

\subsubsection{Oscillatory spiral mode}

For the infinite layer, in the Prandtl number range from 0.14 to 0.45 , the most dangerous mode is the oscillatory spiral mode (rolls with the axis parallel to $z$ and associated with a zero wavenumber $k$ ) related to the development of internal waves in a layer with stable temperature stratification; these waves propagate in the direction perpendicular to the roll axis, i.e. in the $x$ direction in our situation. For a channel width equal to 6.28 , this mode is already strongly stabilized comparatively with the infinite layer case (figure 14a), but it remains the most dangerous in the Prandtl number range from $P r=0.15$ to $P r=0.25$. This mode is still found for $l=4$ (figure 14a), but the critical thresholds are already higher than those obtained for the steady hydrodynamic modes. The dependence of the oscillation angular frequency on 
(a)

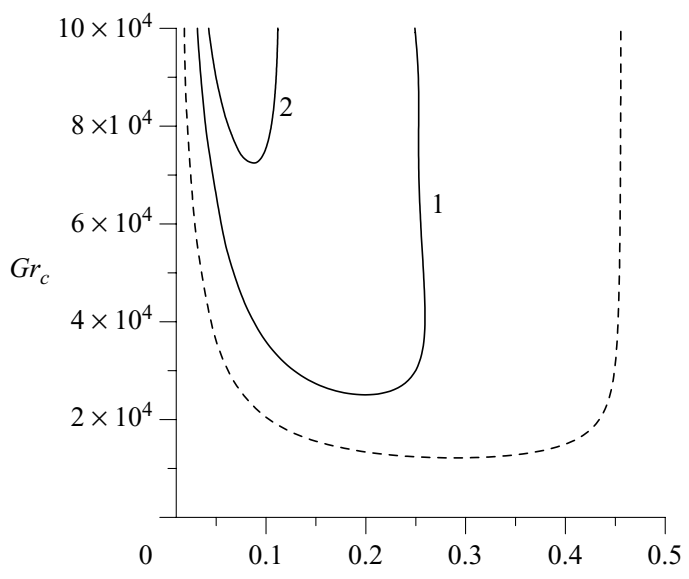

(b)

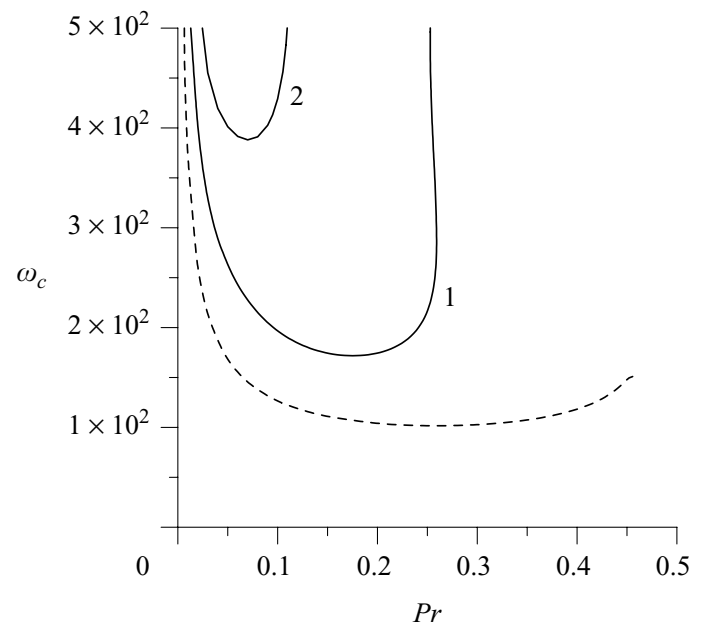

FiguRE 14. Critical Grashof number $G r_{c}(a)$ and angular frequency $\omega_{c}(b)$ versus the Prandtl number $\operatorname{Pr}$ for the oscillatory spiral mode for two channel aspect ratios: $l=6.28(1), l=4(2)$. The dashed line refers to the infinite horizontal layer.

the Prandtl number, displayed in figure 14(b) for the same three cases, $l \rightarrow \infty, l=6.28$ and $l=4$, shows that the frequency increases with the increase of the corresponding critical Grashof number.

A further decrease of $l$ results in the sharp growth of the critical Grashof numbers and oscillation frequencies, the decrease of the Prandtl number range where the oscillatory spiral mode exists, and the shift of this Prandtl number range towards smaller values of $P r$. These behaviours are supported by the results presented in table 2 where, for different values of $l$, the minimum critical Grashof number $G r_{c_{\min }}$, the $\mathrm{Pr}$ location of this minimum $\operatorname{Pr}_{\min }$ and the corresponding angular frequency are given. This table also shows that $G r_{c_{m i n}}$ increases almost vertically when $l$ approaches the value 3.65 , indicating that the oscillatory spiral mode does not exist any more for lower values of $l$. We suggest that the strong stabilization of the oscillatory spiral mode with the decrease of $l$ could be connected to the fact that the sidewalls prevent the natural propagation of the internal waves in the transverse $x$ direction. 


\begin{tabular}{lrrr}
\multicolumn{1}{c}{$l$} & $G r_{c_{\min }}$ & $P r_{\min }$ & $\omega_{\min }$ \\
6.28 & 25234 & 0.198 & 175 \\
6. & 26375 & 0.191 & 185 \\
5.5 & 29506 & 0.175 & 207 \\
5. & 35096 & 0.154 & 242 \\
4.5 & 45965 & 0.126 & 297 \\
4.2 & 58260 & 0.105 & 349 \\
4. & 72409 & 0.087 & 398 \\
3.9 & 83613 & 0.077 & 432 \\
3.8 & 101174 & 0.065 & 480 \\
3.7 & 136533 & 0.052 & 564 \\
3.65 & 183251 & 0.042 & 664
\end{tabular}

TABLE 2. Variation with the aspect ratio $l$ of the minimum critical Grashof number when varying $\operatorname{Pr}$ for the oscillatory spiral mode $G r_{c_{m i n}}$, the $\operatorname{Pr}$ location of this minimum $P r_{\min }$ and the corresponding angular frequency $\omega_{\min }$.

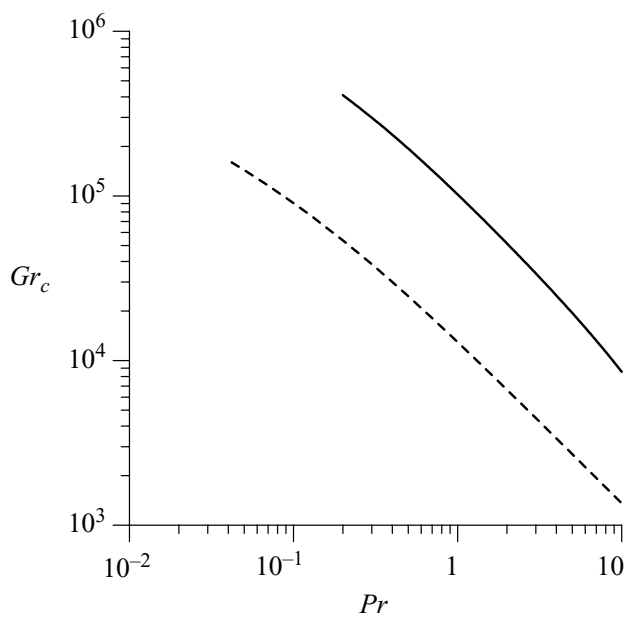

Figure 15. Critical Grashof number $G r_{c}$ versus the Prandtl number $\operatorname{Pr}$ for the steady spiral mode for the channel aspect ratio $l=1$. The dashed line refers to the infinite horizontal layer.

\subsubsection{Steady spiral mode}

In the case of the infinite horizontal layer between rigid conducting plates, at higher values of the Prandtl number the steady spiral instability modes of Rayleigh-type are the most dangerous. The origin of these modes is related to the existence of zones with potentially unstable temperature stratification in the vicinity of the horizontal walls (see figures $4 c$ and $5 c$ ). For the channel with square cross-section $(l=1)$, the results show that at Prandtl numbers higher than 0.3 , the most dangerous modes are also the steady spiral instability modes of Rayleigh-type. These modes are strongly stabilized by the transverse confinement compared to the infinite layer case (figure 15), similarly to what was found for the oscillatory spiral modes. The steady spiral modes, however, do not disappear with the decrease of the channel width $l$ and are still effective for widths smaller than 1 . This can be related to the fact that the Rayleightype perturbations (corresponding to almost square rolls) develop in the relatively thin layers of inverse stratification near the top and bottom of the channel, so that their 
wavelength is quite short, around 0.8 times the height of the channel according to the infinite layer results. This implies that a channel with $l=0.8$ can still accommodate two counter-rotating rolls, and even that a channel with $l=0.4$ can still accommodate one of these rolls. Note finally that with the increase of the channel width, the critical curve for the steady spiral mode is shifted downwards and approaches the critical curve obtained for the infinite layer.

\section{Conclusion}

In this paper, we have studied the steady convective flows arising in horizontal channels of rectangular cross-section submitted to a longitudinal temperature gradient and the stability of these flows with respect to three-dimensional perturbations. The structure of the basic flow in the case of a channel with a finite transverse width differs from the flow structure in the layer of infinite extent. The modifications are qualitative and not only due to the trivial effect of convective flow braking near the channel sidewalls. Namely, at non-zero Prandtl number values, a transverse four-vortex flow arises and leads to the modification of the longitudinal convective flow. These four vortices are generated by the horizontal temperature difference between the sidewalls and the central region of the channel. Indeed, in the upper part of this central region heat is brought by the convective flow from the hot end zone, whereas in the lower part a cooling is induced by a cold convective flow coming from the cold end zone. With the growth of the channel transverse width, the flow in the central part becomes similar to that observed in the infinite layer, and the transverse flow, with only weak changes of intensity, is now concentrated near the sidewalls.

The peculiarities of the structure of the basic flow, uniform along the channel length, predefine to a large extent the behaviour of the small perturbations which develop in this flow. As well as in the case of the infinite layer, at low values of the Prandtl number, the main instability mechanism is of hydrodynamic origin and related to the development of perturbations at the counter stream boundary, in the region where the second derivative of the vertical profile of the basic velocity changes its sign. The increase of the Prandtl number leads to the stabilization of this instability, due to a stable temperature stratification in the region where the perturbations develop. Differences are induced, however, by the finite channel width. First of all, the stabilization occurs earlier since the arising transverse flow leads to the decrease of the longitudinal flow gradients near the channel axis. But the main feature is the appearance of a new instability mode, denoted as sidewall mode. This new hydrodynamic mode develops near the sidewalls because the transverse flow leads to an increase of the shear in these zones compared to the central part of the channel. The stabilization effect with the increase of the Prandtl number is less effective for this mode than for the usual hydrodynamic mode. This is due to the fact that the increase of $\mathrm{Pr}$ also induces the growth of the transverse flow and thus the growth of the shear in the sidewall regions, which counteract the stabilization effect due to the temperature stratification. As a result, the stabilization of this mode occurs more slowly, and at large enough Prandtl numbers just the sidewall mode becomes responsible for the instability. Note that this sidewall mode is obtained for any width of the cross-section, and from this point of view, the infinite layer case is not the limit case for the channel with finite cross-section width. However, one can expect that at large values of the cross-section width, the nonlinear convection developing in the sidewall areas will not penetrate deeply towards the central zone where the situation will thus be close to the case of the infinite layer. 


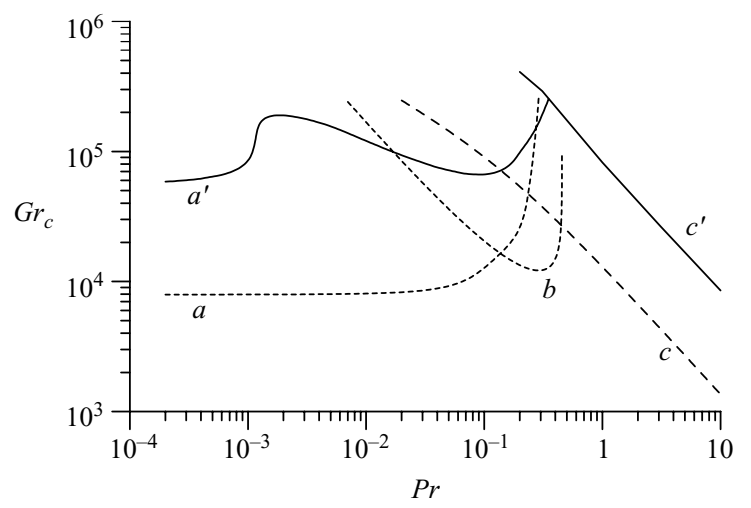

FiguRE 16. Critical Grashof number $G r_{c}$ versus the Prandtl number $\operatorname{Pr}$ for the different instability modes in the whole investigated Prandtl number range: solid lines for the channel aspect ratio $l=1$, dashed lines for the infinite horizontal layer. The labels $a$ and $a$ ' refer to the hydrodynamic mode, the label $b$ to the oscillatory spiral mode and the labels $c$ and $c$ ' to the steady spiral mode.

Concerning the two spiral instabilities which appear for larger Prandtl numbers in the infinite layer case, they still exist for large-width channels, but with critical thresholds substantially increased compared to the infinite layer. When the channel width is further decreased, the thresholds evolve to still larger critical values. This increase of the thresholds, however, is quite continuous for the steady spiral mode, so that this mode can be found even for small-width channels, whereas a very sharp increase is found for the oscillatory spiral mode around $l=3.65$, which corresponds to the disappearance of this mode for smaller channel widths.

The influence of the channel width on the development of the instabilities is summarized in figure 16 where the thresholds for the different instabilities are given for $l \rightarrow \infty$ (infinite layer) and $l=1$ (square cross-section): we clearly see the general stabilization effect as the width is decreased and the evolution of the most dangerous modes with the appearance of the new sidewall hydrodynamic mode and the disappearance of the oscillatory spiral mode.

Further studies concerning the stabilization of these buoyant flows by constant magnetic fields are reported in the companion paper by Lyubimov et al. (2008).

\section{REFERENCES}

Braunsfurth, M. G., Skeldon, A. C., Juel, A., Mullin, T. \& Riley, D. S. 1997 Free convection in liquid gallium. J. Fluid Mech. 342, 295-314.

CARRUthers, J. R. 1977 Thermal convection instabilities relevant to crystal growth from liquids. In Preparation and Properties of Solid State Materials (ed. W. R. Wilcox \& R. A. Lefever), vol. 3, pp. 1-121. Marcel Dekker.

Cormack, D. E., Leal, L. G. \& Imberger, J. 1974 Natural convection in a shallow cavity with differentially heated endwalls. Part1. Asymptotic theory. J. Fluid Mech. 65, 209-229.

Gelfgat, A. Y., Bar-Yoseph, P. Z. \& Yarin, A. L. 1999 Stability of multiple steady states of convection in laterally heated cavities. J. Fluid Mech. 388, 315-334.

Gershuni, G. Z., Zhukhovitskit, E. M. \& Myznikov, V. M. 1974a Stability of a plane-parallel convective flow of a liquid in a horizontal layer. J. Appl. Mech. Tech. Phys. 15 (1), 78-82.

Gershuni, G. Z., Zhukhovitskit, E. M. \& Myznikov, V. M. $1974 b$ Stability of plane-parallel convective fluid flow in a horizontal layer relative to spatial perturbations. J. Appl. Mech. Tech. Phys. 15 (5), 706-708. 
Gill, A. E. 1974 A theory of thermal oscillations in liquid metals. J. Fluid Mech. 64, 577-588.

Hart, J. E. 1972 Stability of thin non-rotating Hadley circulations. J. Atmos. Sci. 29, 687-697.

Hart, J. E. 1983 A note on the stability of low-Prandtl-number Hadley circulations. J. Fluid Mech. 132, 271-281.

Henry, D. \& Ben Hadid, H. 2007a Multiple flow transitions in a box heated from the side in low-Prandtl-number fluids. Phys. Rev. E 76, 016314(1-9).

Henry, D. \& Ben Hadid, H. $2007 b$ Multiple modes of instability in a box heated from the side in low-Prandtl-number fluids. Phys. Fluids 19, 081702(1-4).

Henry, D. \& Buffat, M. 1998 Two- and three-dimensional numerical simulations of the transition to oscillatory convection in low-Prandtl number fluids. J. Fluid Mech. 374, 145-171.

Henry, D., Juel, A., Ben Hadid, H. \& Kaddeche, S. 2008 Directional effect of a magnetic field on oscillatory low-Prandtl-number convection. Phys. Fluids 20, 034104(1-12).

Hof, B., Juel, A., Zhao, L., Henry, D., Ben Hadid, H. \& Mullin, T. 2004 On the onset of oscillatory convection in molten gallium. J. Fluid Mech. 515, 391-413.

Hung, M. C. \& ANDERECK, C. D. 1990 Subharmonic transition in a moderately shallow cavity. In Numerical Simulation of Oscillatory Convection in Low-Pr Fluids (ed. B. Roux), GAMM Workshop, Notes on Numerical Fluid Mechanics, vol. 27, pp. 338-343. Vieweg.

Hurle, D. T. J., Jakeman, E. \& Johnson, C. P. 1974 Convective temperature oscillations in molten gallium. J. Fluid Mech. 64, 565-576.

Kaddeche, S., Henry, D. \& Ben Hadid, H. 2003 Magnetic stabilization of the buoyant convection between infinite horizontal walls with a horizontal temperature gradient. J. Fluid Mech. 480, $185-216$

Kuo, H. P. \& Korpela, S. A. 1988 Stability and finite amplitude natural convection in a shallow cavity with insulated top and bottom and heated from a side. Phys. Fluids 31, 33-42.

LAURE, P. 1987 Study on convective motions in a rectangular cavity with a horizontal gradient of temperature. J. Méc. Théor. Appl. 6, 351-382.

Laure, P. \& Roux, B. 1987 Synthesis of the results obtained by a stability analysis of the convective motions in a horizontal cavity of large extent. C. R. Acad. Sci. Paris 305, 1137-1143.

Lyubimov, D. V., Lyubimova, T. P. \& Morozov, V. A. 2001 Software package for numerical investigation of linear stability of multidimensional flows. Bull. Perm Univ.: Inf. Syst. Technol. 5, 74-81.

Lyubimov, D. V., Lyubimova, T. P., Perminov, A. B., Henry, D. \& Ben Hadid, H. 2009 Stability of convection in a horizontal channel subjected to a longitudinal temperature gradient. Part 2. Effect of a magnetic field. J. Fluid Mech. 635, 297-319.

Roux, B. ed. 1990 Numerical simulation of oscillatory convection in low-Pr fluids. GAMM Workshop, Notes on Numerical Fluid Mechanics, vol. 27. Vieweg.

Roux, B., Ben Hadid, H. \& LaURe, P. 1989a Hydrodynamical regimes in metallic melts subject to a horizontal temperature-gradient. Eur. J. Mech. B/Fluids 8, 375-396.

Roux, B., Ben Hadid, H. \& Laure, P. $1989 b$ Numerical simulation of oscillatory convection in semiconductor melts. J. Cryst. Growth 97, 201-216.

Skeldon, A. C., Riley, D. C. \& Cliffe, K. A. 1996 Convection in a low Prandtl number flufid. J. Cryst. Growth 162, 95-106.

Touihri, R., Ben Hadid, H. \& Henry, D. 1999 On the onset of convective instabilities in cylindrical cavities heated from below. I. Pure thermal case. Phys. Fluids 11, 2078-2088.

Vaux, S., Ben Hadid, H. \& Henry, D. 2006 Study of the hydrodynamic instabilities in a differentially heated horizontal circular cylinder corresponding to a Bridgman growth configuration. J. Cryst. Growth 290, 674-682.

WAKITANI, S. 2001 Numerical study of three-dimensional oscillatory natural convection at low Prandtl number in rectangular enclosures. J. Heat Transfer 123, 77-83.

WinTERS, K. H. 1988 Oscillatory convection in liquid metals in a horizontal temperature gradient. Intl J. Numer. Meth. Engng 25, 401-414. 